\title{
Calculating Dispersion Relations for Waveguide Immersed in Perfect Fluid
}

\section{Audrius Nečiūnas ${ }^{a}$, Martynas Patašius ${ }^{a, b}$ and Rimantas Barauskas ${ }^{a}$}

\author{
${ }^{a}$ Kaunas University of Technology, Department of Applied Informatics \\ Studentu str. 50, Kaunas, Lithuania \\ ${ }^{b}$ Kaunas University of Technology, Institute of Biomedical Engineering \\ K. Baršausko g. 59, Kaunas, Lithuania \\ E-mail(corresp.): Audrius.Neciunas@ktu.lt \\ E-mail: Martynas.Patasius@ktu.lt \\ E-mail: Rimantas.Barauskas@ktu.1t
}

Received November 11, 2017; revised March 19, 2018; accepted March 20, 2018

\begin{abstract}
Conventional finite element method (FEM) is capable of obtaining wave solutions, but large discretized structures at high frequency require high computational resources, the computational domain can be reduced by combining FEM with analytical assumption for guided wave. Semi Analytical Finite Element (SAFE) formulation for immersed waveguide in perfect fluid is used for acquiring propagating wave modes as dynamic equilibrium states. Modes are solutions to eigenvalue problem and provide with important characteristic features of the guided waves - phase velocity, attenuation, wave structure, etc. The effect of surrounding leaky medium is modeled via traction boundary condition, which is based on assumption of the continuity of stresses at solid-fluid interface. The boundary condition causes wave attenuation due to energy leakage into outer medium. The derivation of the eigenproblem takes into account complex wavenumbers of leaky wave in fluid and guided wave in a three-dimensional waveguide. Linearization procedure for solving nonlinear eigenvalue problem is used. Dispersion relations for immersed waveguide with Rayleigh damping are obtained. The limits of applications of Rayleigh damping and convergence analysis of immersed waveguide model are discussed.
\end{abstract}

Keywords: semi analytical finite element, eigenvalue problem, complex wavenumber.

AMS Subject Classification: 74S05.

Copyright (c) 2018 The Author(s). Published by VGTU Press

This is an Open Access article distributed under the terms of the Creative Commons Attribution License (http://creativecommons.org/licenses/by/4.0/), which permits unrestricted use, distribution, and reproduction in any medium, provided the original author and source are credited. 


\section{Introduction}

Periodic loading on the structure causes its dynamic response as an elastic wave. Guided waves can be widely applied in various fields such as non-destructive testing (NDT) [3]. Any structure, which is uniform (both in geometrical and physical sense) in at least one direction, can be regarded as a waveguide. The simplest waveguides are plates, rods, beams, etc. Important features of guided waves are dispersion relations. They provide information about wavenumbers, group and phase velocities, displacement patterns of the waves, etc. and can be retrieved analytically just in the cases of simplest waveguides [5,22].

Obtaining dispersion characteristics in complex structures requires a different numerical approach most often based on finite element method (FEM) [24]. FEM is robust and flexible tool widely preferred for solving general problems for structures of generic geometry and material setup. However, this approach becomes unsuitable as it requires a lot of computation resources when large structures at high frequencies are considered. The size of FE models of threedimensional (3D) waveguides can be simplified by taking just a small representative portion of the waveguide structure with the periodicity condition along the waveguide. This enables to obtain the wave characteristics via eigenproblem, where the whole number of wavelengths is contained in the length of the structure. This technique is used in wave and finite element method (WFEM) and is easily adopted in commercial FE software by optimizing computational resources for simulation of propagating waves [17]. Another approach based on semi-analytical method is Scaled Boundary Finite Element Method (SBFEM) [10]. The Semi Analytical Finite Element Method (SAFE) requires discretization of the cross-section of the waveguide [12,23]. The common assumption for WFEM, SBFEM and SAFE as semi analytical techniques considers the travelling wave displacement field as separable by using harmonic term $e^{-i k z}$ along the axis of the waveguide infinite in $O Z$ direction.

More general and realistic propagation scenario includes several possible damping mechanisms resulting in amplitude attenuation. The decay of elastic wave may be caused by material damping, fluid viscosity or by energy leakage into the infinite medium surrounding the waveguide [16]. Analysis of damped waveguides involves complex numbers into eigenproblem formulation. Viscoelasticity is modeled by using hysteretic or Kelvin Voigt approaches, or by introducing complex-valued Young's modulus [2,21]. Fluid viscosity must be taken into account in case the waveguide interacts with in the fluid domain. A hypothetical isotropic solid can be used for representation of the fluid accommodating viscosity [15]. The attenuation due to energy leakage affects both elastic and viscoelastic waveguide.

While there are many works addressed to waveguides in vacuum with stressfree condition, the techniques, that support interaction with leaky medium, are still under development. The problems including concrete rods buried in soil or thin walled pipes (hollow cylinders) filled with fluid require modeling the surrounding leaky medium, which causes propagating wave to attenuate due to leakage of the energy. A common way to simulate the wave transmission to surrounding infinite medium by FEM requires to eliminate or to 
significantly reduce the reflections from the boundaries of the computational domain via absorbing regions around the waveguide immersed/imbedded in simulated leaky medium [4]. As the travelling wave in surrounding medium approaches boundaries of medium's domain, it gradually disappears. However, this external domain requires the number of finite elements many times bigger than the number of finite elements in the waveguide. The perfectly matched layer (PML) approach is similar to the technique of absorbing layers, but the domain representing medium and high attenuating region remains [20]. Further additional sorting procedure is necessary afterwards in order to keep only physically meaningful solutions. The $2.5 \mathrm{D}$ boundary element technique was used to model the surrounding leaky medium. This approach involves complex non-linear eigen problem for obtaining wavenumbers for damped waveguide, however computational time for this technique is greater in comparison to SAFE formulation coupled to infinite element or PML [14]. As demonstrated, the simple approximation by dashpot boundary conditions included in SBFEM can lead to sufficiently accurate results for waveguides embedded in surrounding solid medium [8]. An iterative exact dashpot boundary condition was employed in SBFEM for obtaining the wavenumbers for waveguide immersed in perfect fluid [7], Hayashi et al has extended SAFE formulation for plates loaded with leaky medium [11]. Waveguides interacting with fluid support distinct quasi-Scholte surface waves, which are absent in case of vacuum. Scholte wave propagates at the interference between the waveguide and fluid and tends to dissipate energy rather slowly.

This study adopts the approach of immersed plate for acquiring dispersion relations for three-dimensional waveguide of rectangular cross-section immersed in perfect fluid using SAFE procedure. The used damping mechanism for waveguide combines leaky waves and material damping. The attenuation due to energy leakage is considered in this study, as it does not require modeling of outer leaky medium in case of perfect fluid. The theoretical investigation of the impact of Rayleigh damping to dispersion relations for immersed waveguide is carried out.

\section{Traction condition for immersed waveguide in perfect fluid}

\subsection{SAFE formulation for traction-free waveguide}

In this section the waveguide in vacuum will be described and the outer medium, a perfect fluid, will be discussed in the next section. SAFE approach assumes that the waveguide with arbitrary cross-section has no geometrical variation along at least one axis. Therefore, only the cross-section has to be discretized using FE. A rectangular bar with cross-section parallel to $X O Y$ plane and infinite along $O Z$ direction is considered in this study. Hamilton's virtual work principle for discretized structure in vacuum yields the eigen problem as

$$
\left(\mathbf{K}_{1}+i k_{Z} \mathbf{K}_{2}+k_{Z}^{2} \mathbf{K}_{3}+\omega^{2} \mathbf{M}\right) \mathbf{U}=\mathbf{0} .
$$

The solution of (2.1) provides wavenumbers $k_{Z}$ as eigenvalues and $\mathbf{U}$ am- 
plitudes of nodal displacements as eigenvectors of wave solution at given real valued angular frequency $\mathbf{U}$. The matrices for a single FE involved in eigenvalue problem (2.1) are defined as

$$
\begin{aligned}
& \mathbf{M}_{e}=\iint \mathbf{N}(x, y)^{T} \rho_{e} \mathbf{N}(x, y) d x d y, \quad \mathbf{K}_{1 e}=\iint \mathbf{B}_{1}^{T} \mathbf{D}_{e} \mathbf{B}_{1} d x d y \\
& \mathbf{K}_{2 e}=\iint\left(\mathbf{B}_{1}^{T} \mathbf{D}_{e} \mathbf{B}_{2}-\mathbf{B}_{2}^{T} \mathbf{D}_{e} \mathbf{B}_{1}\right) d x d y, \quad \mathbf{K}_{3 e}=\iint \mathbf{B}_{2}^{T} \mathbf{D}_{e} \mathbf{B}_{2} d x d y,
\end{aligned}
$$

where $T$ denotes matrix transform operator, $\mathbf{N}$ is the matrix of shape functions, $\rho$ is the mass density, $\mathbf{D}$ is elasticity matrix, $\mathbf{B}_{1}$ and $\mathbf{B}_{2}$ are strain matrices containing partial derivatives of shape functions. As a result, the displacement in spatial and time domain reads as

$$
\mathbf{U}(x, y, z, t)=\mathbf{U}(x, y) e^{i(k z-\omega t)},
$$

which indicates that the displacement field is expressed via harmonic wave propagating in $O Z$ direction.

In this way, SAFE formulation exploits harmonic spatial and temporally exponential function $e^{i(k z-\omega t)}$ to numerically reduce the three-dimensional model to two-dimensional one.

\subsection{Traction condition for the waveguide}

Let's consider a waveguide that has infinite length in $O Z$ direction, immersed in infinite leaky medium, assumed to be perfect non-viscous fluid, which does not support shear waves (Figure 1(a)).

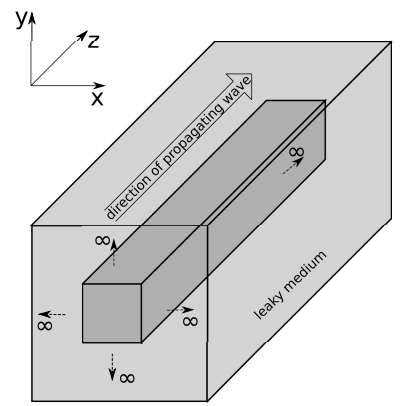

(a)

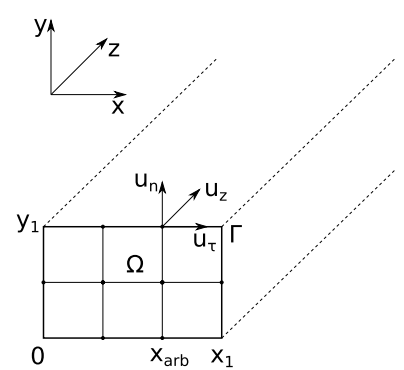

(b)

Figure 1. (a) Scheme of immersed waveguide; (b) Discretization of cross-section of the waveguide.

The guided wave is assumed to be travelling in a waveguide along $O Z$ direction with wavenumber vector $\mathbf{k}_{z}$ (direction of which coincides with $O Z$ axis) at angular frequency $\omega$. SAFE approach represents the finite element domain $\Omega$ as discretized cross-section of the waveguide and is a boundary perimeter of the cross-section (Figure 1(b)). The coordinate system of the structure is oriented in such a way that the directions of nodal displacements 1) $u_{\tau}$ tangential to 
surface of cross-section, 2) $u_{n}$ normal to surface of cross-section, 3$) u_{z}$ parallel to waveguide axis on upper side of $\Gamma$ respectively match $O X$ or $O Y$ and $O Z$ global directions depending on the concerned side of waveguide. Displacements $u_{\tau}$ and $u_{z}$ would excite shear waves in surrounding leaky medium. In case of the perfect fluid only displacement $u_{n}$ excites the longitudinal wave in outer fluid.

The traveling wave in the waveguide also generates the pressure wave in the surrounding fluid. This pressure wave can be found as a superposition of two separate waves traveling in the directions of waveguide axes $O Z$ and $u_{n}$ respectively. The wave in the fluid is characterized by wavenumber vector $\tilde{\mathbf{k}}$. The norm of the wavenumber vector associated with this pressure wave is related with acoustic properties of the leaky medium as

$$
\tilde{k}=\omega / \tilde{c}_{L}
$$

where tilde superscript denotes that the parameter corresponds to the leaky medium.

The direction of wavenumber vector $\tilde{\mathbf{k}}$ reads as

$$
\tilde{k}^{2}=\tilde{k}_{L}^{2}+\tilde{k}_{z}^{2},
$$

where $\tilde{k}_{L}$ is the projection of $\tilde{\mathbf{k}}$ in plane perpendicular to the cross-section of the waveguide, $\tilde{k}_{z}$ is the projection of $\tilde{\mathbf{k}}$ on axis $O Z$ and coincides with the wavenumber of travelling wave in the waveguide, i.e., $\tilde{k}_{z}=k_{z}$ (Figure $2(\mathrm{a})$ ). In this work the analysis is restricted to convex cross-sections in order to avoid travelling wavefield back to the waveguide surface. Let $\Gamma 1$ be the segment

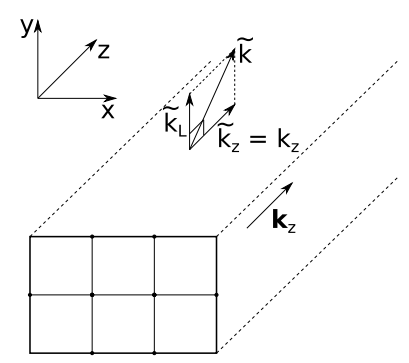

(a)

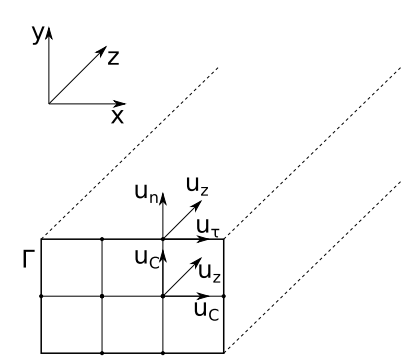

(b)

Figure 2. (a) Wavenumbers at interface of waveguide and fluid; (b) schematic classification of nodal displacements in the cross-section.

of the cross-section perimeter determined between two points $0, y 1$ and $x 1, y 1$ (Figure 1(b)). Let $x_{a r b}$ being on $\Gamma 1$ to coincide with FE nodes with respect to $O X$ axis, while following the assumption, that stress and displacements are continuous at interface of solid and fluid. That yields waveguide surface traction caused by fluid:

$$
\mathbf{t}_{\Gamma 1}=\left(\begin{array}{c}
t_{n} \\
t_{\tau} \\
t_{z}
\end{array}\right)=\mathbf{N} \mathbf{T}_{j} e^{i k_{z} z},
$$


where $\mathbf{N}$ is the shape function with constraint $y=y 1, j=\overline{1, m},(m-1)$ is the number of FEs along boundary $\Gamma 1, \mathbf{T}_{j}$ is nodal external traction vector at waveguide boundary as:

$$
\mathbf{T}_{j}=\left(\begin{array}{c}
t_{n} \\
0 \\
0
\end{array}\right)=-i \frac{\omega^{2}}{\tilde{k}_{L}}\left(\begin{array}{ccc}
\tilde{\rho} & 0 & 0 \\
0 & 0 & 0 \\
0 & 0 & 0
\end{array}\right)\left(\begin{array}{l}
u_{n} \\
u_{\tau} \\
u_{z}
\end{array}\right)=-i \frac{\omega^{2}}{\tilde{k}_{L}} \mathbf{C}\left(\begin{array}{l}
u_{n} \\
u_{\tau} \\
u_{z}
\end{array}\right) .
$$

The surface traction for waveguide (2.4) accounts for the load caused by fluid, which is the function of acoustic properties of the fluid, angular frequency of the travelling wave and displacements at the boundary of solid and fluid. A detailed derivation of (2.3) is given in Appendix.

\section{Obtaining dispersion relations}

\subsection{Nonlinear eigenvalue problem}

The external boundary condition must be included into the general eigenvalue problem as

$$
\left(\mathbf{K}_{1}+i k_{Z} \mathbf{K}_{2}+k_{Z}^{2} \mathbf{K}_{3}-\omega^{2} \mathbf{M}\right) \mathbf{u}=\mathbf{F}_{\text {fluid }}+\mathbf{F}_{\text {loading }},
$$

where $\mathbf{F}_{\text {fluid }}$ is the nodal force vector due to fluid traction condition, $\mathbf{F}_{\text {loading }}$ is the nodal force vector due to external dynamic loading in case the forced wave response is concerned. In case $\mathbf{F}_{\text {loading }}=0,(3.1)$ is treated as an eigenvalue problem, the solution of which gives dispersion relations for waveguide immersed in fluid.

$\mathbf{F}_{\text {fluid }}$ summarizes the external traction caused by fluid loading:

$$
\mathbf{F}_{\text {fluid }}=i \frac{\omega^{2}}{\tilde{k}_{L}} \mathbf{Q u}=i \frac{\omega^{2}}{\tilde{k}_{L}} \mathbf{Q} \mathbf{U} e^{-i k_{z} z},
$$

where coefficient matrix $\mathbf{Q}$ characterizes the distribution of fluid tractions among the nodes on surface perimeter $\Gamma$ as in [8]:

$$
\mathbf{Q}=\int_{s \in \Gamma}(\mathbf{N}(s))^{T} \mathbf{C N}(s) d s
$$

where $\mathbf{N}(s)$ is one-dimensional shape function used to interpolate $x$ and $y$ coordinates on $\Gamma, s$ is a circumferential integral coordinate running along $\Gamma$.

Finally, the governing equation for immersed waveguides is obtained:

$$
\left(\mathbf{K}_{1}+i k_{Z} \mathbf{K}_{2}+k_{Z}^{2} \mathbf{K}_{3}-\omega^{2} \mathbf{M}-i \frac{\omega^{2}}{\tilde{k}_{L}} \mathbf{Q}\right) \mathbf{U}=\mathbf{0} .
$$

\subsection{Solving eigenvalue problem}

In this study (3.2) is solved by extending the technique proposed in paper [11] to three-dimensional waveguide. Two stage procedure of algebraic transformation 
and linearization of (3.2) is employed. The $m$-th wavemode $\left(k_{Z m}, \tilde{k}_{L m}, \boldsymbol{\varphi}_{m}\right)$ must satisfy equation:

$$
\left(\mathbf{K}_{1}+i k_{Z m} \mathbf{K}_{2}+k_{Z m}^{2} \mathbf{K}_{3}-\omega^{2} \mathbf{M}-i \frac{\omega^{2}}{\tilde{k}_{L m}} \mathbf{Q}\right) \boldsymbol{\varphi}_{m}=\mathbf{0} .
$$

Further transformation is based on symmetry of wave solutions with respect to $X O Y$ plane. Identical waves propagating in positive and negative $O Z$ directions are described by (3.3). In order to exploit this feature, we split eigen vector $\varphi_{m}$ with respect of displacements in directions of $O Z$ and other than $O Z$ :

$$
\boldsymbol{\varphi}_{m}=\left(\begin{array}{c}
\boldsymbol{\varphi}_{o m} \\
\boldsymbol{\varphi}_{z m}
\end{array}\right) .
$$

For convenience of post-processing of modal results eigen vector $\varphi_{m}$ is split by separating nodal degrees of freedom (dof) with respect to displacements $u_{n}$ on $\Gamma$, that correspond to the longitudinal wave in the fluid (denoted by L), displacements $u_{\tau}$ on $\Gamma$, that would excite shear wave (denoted by $\mathrm{S}$ ), displacements $u_{z}$ along $O Z$ axis on entire cross-section (denoted by $z$ ) and introduced displacements $u_{C}$ in the core of cross-section (denoted by C) (Figure 2(b)).

The preferable reordering of dof could be summarized as

$$
\boldsymbol{\varphi}_{m}=\left(\begin{array}{c}
\boldsymbol{\varphi}_{o m} \\
\boldsymbol{\varphi}_{z m}
\end{array}\right)=\left(\boldsymbol{\varphi}_{L m}, \boldsymbol{\varphi}_{S m}, \boldsymbol{\varphi}_{C m}, \boldsymbol{\varphi}_{z m}\right)^{T} .
$$

The matrices in (3.3) are divided into block components in accordance with (3.4) resulting in

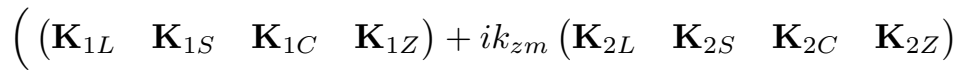

$$
\begin{aligned}
& +k_{z m}^{2}\left(\begin{array}{llllllll}
\mathbf{K}_{3 L} & \mathbf{K}_{3 S} & \mathbf{K}_{3 C} & \left.\mathbf{K}_{3 Z}\right)-\omega^{2}\left(\mathbf{M}_{L}\right. & \mathbf{M}_{S} & \mathbf{M}_{C} & \mathbf{M}_{Z}
\end{array}\right) \\
& \left.-i \frac{\omega^{2}}{\tilde{k}_{L m}}\right)\left(\boldsymbol{\varphi}_{L m}, \boldsymbol{\varphi}_{S m}, \boldsymbol{\varphi}_{C m}, \boldsymbol{\varphi}_{z m}\right)^{T}=\mathbf{0} \text {. }
\end{aligned}
$$

Symmetrical solution with respect to $O Z$ direction means that displacement distribution with $-k_{Z m}$ wavenumber and corresponding eigenvector $\left(\begin{array}{c}\boldsymbol{\varphi}_{o m} \\ -\boldsymbol{\varphi}_{z m}\end{array}\right)$ also satisfies equation:

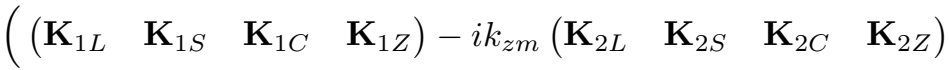

$$
\begin{aligned}
& +k_{z m}^{2}\left(\begin{array}{lllllll}
\mathbf{K}_{3 L} & \mathbf{K}_{3 S} & \mathbf{K}_{3 C} & \left.\mathbf{K}_{3 Z}\right)-\omega^{2}\left(\mathbf{M}_{L}\right. & \mathbf{M}_{S} & \mathbf{M}_{C} & \mathbf{M}_{Z}
\end{array}\right) \\
& \left.-i \frac{\omega^{2}}{\tilde{k}_{L m}}-i \omega^{2}\left(\mathbf{Q}_{L} \quad \mathbf{Q}_{S} \quad \mathbf{Q}_{C} \quad \mathbf{Q}_{Z}\right)\right)\left(\boldsymbol{\varphi}_{L m}, \boldsymbol{\varphi}_{S m}, \boldsymbol{\varphi}_{C m},-\boldsymbol{\varphi}_{z m}\right)^{T}=\mathbf{0} .
\end{aligned}
$$

Linear combination of $(3.5)$ and $(3.6)-((3.5)+(3.6)) / 2+k_{z m}((3.5)-(3.6)) / 2$ - provides the eigenvalue problem as

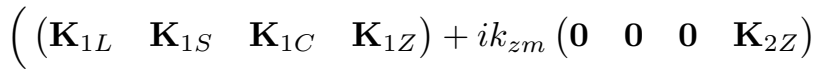

$$
\begin{aligned}
& +k_{z m}^{2}\left(\begin{array}{lllllll}
\mathbf{K}_{3 L} & \mathbf{K}_{3 S} & \mathbf{K}_{3 C} & \left.\mathbf{K}_{3 Z}\right)-\omega^{2}\left(\mathbf{M}_{L}\right. & \mathbf{M}_{S} & \mathbf{M}_{C} & \mathbf{M}_{Z}
\end{array}\right) \\
& \left.-i \frac{\omega^{2}}{\tilde{k}_{L m}}\left(\begin{array}{llll}
\mathbf{Q}_{L} & \mathbf{Q}_{S} & \mathbf{Q}_{C} & \mathbf{Q}_{Z}
\end{array}\right)\right)\left(\boldsymbol{\varphi}_{L m}, \boldsymbol{\varphi}_{S m}, \boldsymbol{\varphi}_{C m}, k_{z m} \boldsymbol{\varphi}_{z m}\right)^{T}=\mathbf{0},
\end{aligned}
$$


which can be further simplified to

$$
\left(\mathbf{H}_{11}+k_{z m}^{2} \mathbf{H}_{12}+\frac{\mathbf{H}_{0}}{\tilde{k}_{L m}}\right)\left(\boldsymbol{\varphi}_{L m}, \boldsymbol{\varphi}_{S m}, \boldsymbol{\varphi}_{C m}, k_{z m} \boldsymbol{\varphi}_{z m}\right)^{T}=\mathbf{0},
$$

where:

$$
\begin{aligned}
& \mathbf{H}_{11}=\left(\begin{array}{llllll}
\mathbf{K}_{1 L} & \mathbf{K}_{1 S} & \mathbf{K}_{1 C} & \mathbf{K}_{1 Z}
\end{array}\right)-\omega^{2}\left(\begin{array}{llll}
\mathbf{M}_{L} & \mathbf{M}_{S} & \mathbf{M}_{C} & \mathbf{M}_{Z}
\end{array}\right) \\
& \mathbf{H}_{12}=\left(\begin{array}{lllll}
i \mathbf{K}_{2 L}+\mathbf{K}_{3 L} & i \mathbf{K}_{2 S}+\mathbf{K}_{3 S} & i \mathbf{K}_{2 C}+\mathbf{K}_{3 C} & i \mathbf{K}_{2 Z}+\mathbf{K}_{3 Z}
\end{array}\right) \\
& \mathbf{H}_{0}=-i \omega^{2}\left(\begin{array}{lllll}
\mathbf{Q}_{L} & \mathbf{Q}_{S} & \mathbf{Q}_{C} & \mathbf{Q}_{Z}
\end{array}\right)
\end{aligned}
$$

As the mechanical energy of waveguide is transferred to the fluid in normal direction only, the matrix block $\mathbf{H}_{0}$ in (3.8) can be simplified to the form

$$
\mathbf{H}_{0}=-i \omega^{2}\left(\mathbf{Q}_{L} \quad \mathbf{0} \quad \mathbf{0} \quad \mathbf{0}\right) .
$$

By substituting (2.2) to (3.7) we obtain

$$
\left(\mathbf{H}_{1}+k_{L m}^{2} \mathbf{H}_{3}+\frac{\mathbf{H}_{0}}{\tilde{k}_{L m}}\right)\left(\boldsymbol{\varphi}_{L m}, \boldsymbol{\varphi}_{S m}, \boldsymbol{\varphi}_{C m}, k_{z m} \boldsymbol{\varphi}_{z m}\right)^{T}=\mathbf{0}
$$

where $\mathbf{H}_{1}=\mathbf{H}_{11}+\tilde{k}^{2} \mathbf{H}_{12}, \mathbf{H}_{3}=-\mathbf{H}_{12}$. Finally, (3.9) can be rewritten as the 3rd order polynomial eigenvalue problem:

$$
\begin{gathered}
\left(k_{L m} \mathbf{H}_{1}+k_{L m}^{3} \mathbf{H}_{3}+\mathbf{H}_{0}\right)\left(\boldsymbol{\varphi}_{L m}, \boldsymbol{\varphi}_{S m}, \boldsymbol{\varphi}_{C m}, k_{z m} \boldsymbol{\varphi}_{z m}\right)^{T} \\
=\left(k_{L m} \mathbf{H}_{1}+k_{L m}^{3} \mathbf{H}_{3}+\mathbf{H}_{0}\right) \boldsymbol{\varphi}_{m}^{\prime}=\mathbf{0}
\end{gathered}
$$

(3.10) now can be recast to linear eigenvalue problem as

$$
\begin{gathered}
\left(\left(\begin{array}{ccc}
\mathbf{0} & \mathbf{H}_{1} & \mathbf{H}_{0} \\
\mathbf{I} & \mathbf{0} & \mathbf{0} \\
\mathbf{0} & \mathbf{I} & \mathbf{0}
\end{array}\right)-\tilde{k}_{L m}\left(\begin{array}{ccc}
-\mathbf{H}_{3} & \mathbf{0} & \mathbf{0} \\
\mathbf{0} & \mathbf{I} & \mathbf{0} \\
\mathbf{0} & \mathbf{0} & \mathbf{I}
\end{array}\right)\right)\left(\begin{array}{c}
\tilde{k}_{L m}^{2} \boldsymbol{\varphi}_{m}^{\prime} \\
\tilde{k}_{L m} \boldsymbol{\varphi}_{m}^{\prime} \\
\boldsymbol{\varphi}_{m}^{\prime}
\end{array}\right) \\
=\left(\mathbf{A}-\tilde{k}_{L m} \mathbf{B}\right)\left(\tilde{k}_{L m}^{2} \boldsymbol{\varphi}_{m}^{\prime}, \tilde{k}_{L m} \boldsymbol{\varphi}_{m}^{\prime}, \boldsymbol{\varphi}_{m}^{\prime}\right)^{T}=\mathbf{0}
\end{gathered}
$$

where $\mathbf{I}$ is the identity matrix of dimensions $N \times N$, where $N$ is the number of dof over the waveguide cross-section.

(3.11) can be reduced by omitting zero members as:

$$
\begin{array}{r}
\left(\left(\begin{array}{ccc}
\mathbf{0} & \mathbf{H}_{1} & \mathbf{H}_{0}^{\prime} \\
\mathbf{I} & \mathbf{0} & \mathbf{0} \\
\mathbf{0} & \mathbf{I}^{\prime} & \mathbf{0}
\end{array}\right)-\tilde{k}_{L m}\left(\begin{array}{ccc}
-\mathbf{H}_{3} & \mathbf{0} & \mathbf{0} \\
\mathbf{0} & \mathbf{I} & \mathbf{0} \\
\mathbf{0} & \mathbf{0} & \mathbf{I}^{\prime \prime}
\end{array}\right)\right)\left(\begin{array}{c}
\tilde{k}_{L m}^{2} \boldsymbol{\varphi}_{m}^{\prime} \\
\tilde{k}_{L m} \boldsymbol{\varphi}_{m}^{\prime} \\
\boldsymbol{\varphi}_{m}^{\prime \prime}
\end{array}\right) \\
=\left(\mathbf{A}^{\prime}-\tilde{k}_{L m} \mathbf{B}^{\prime}\right)\left(\tilde{k}_{L m}^{2} \boldsymbol{\varphi}_{m}^{\prime}, \tilde{k}_{L m} \boldsymbol{\varphi}_{m}^{\prime}, \boldsymbol{\varphi}_{m}^{\prime \prime}\right)^{T}=\mathbf{0}
\end{array}
$$

where matrices $\mathbf{I}^{\prime}, \mathbf{I}^{\prime \prime}$ and $\mathbf{H}_{0}^{\prime}$ are matrices $\mathbf{I}$ and $\mathbf{H}_{0}$ with last rows and columns removed as necessary. Consequently, $\boldsymbol{\varphi}_{m}^{\prime \prime}$ has as many rows, as $\mathbf{Q}_{L}$, that is, as many, as there are dofs in contact with the fluid. 
It is convenient to present (3.12) as the standard eigenvalue problem:

$$
\left(\left(\mathbf{B}^{\prime}\right)^{-1} \mathbf{A}^{\prime}-\tilde{k}_{L m} \mathbf{I}\right)\left(\begin{array}{c}
\tilde{k}_{L m}^{2} \boldsymbol{\varphi}_{m}^{\prime} \\
\tilde{k}_{L m} \boldsymbol{\varphi}_{m}^{\prime} \\
\boldsymbol{\varphi}_{m}^{\prime \prime}
\end{array}\right)=\left(\left(\mathbf{B}^{\prime}\right)^{-1} \mathbf{A}^{\prime}-\tilde{k}_{L m} \mathbf{I}\right) \mathbf{Y}=\mathbf{0} .
$$

The fluid load can be taken into account in cases of both elastic and viscoelastic waveguide materials. Rheological model of linear viscoelasticity is expressed by using complex Young's modulus in the generalized Maxwell model. This study considers Rayleigh damping in order to account for the linear viscoelasticity of the waveguide under harmonic excitation. Though Rayleigh damping has no unambiguous physical interpretation, it is widely applied due to its convenient mathematical formulation. Rayleigh damping matrix $\mathbf{C}$ is produced from mass and stiffness matrices ( $\mathbf{M}$ and $\mathbf{K})$ :

$$
\mathbf{C}=a \mathbf{M}+b \mathbf{K}
$$

where $a$ and $b$ are scalar coefficients. Substitution of matrix $\mathbf{C}$ into (3.2) yields $[1]$ :

$$
\left(\hat{\mathbf{K}}_{1}+i k_{Z} \hat{\mathbf{K}}_{2}+k_{Z}^{2} \hat{\mathbf{K}}_{3}-\omega^{2} \hat{\mathbf{M}}-i \frac{\omega^{2}}{\tilde{k}_{L}} \mathbf{Q}\right) \mathbf{U}=\mathbf{0}
$$

here

$$
\hat{\mathbf{K}}_{j}=(1-i \omega b) \mathbf{K}_{j}, \quad j=1,2,3, \quad \hat{\mathbf{M}}=\left(1-i \omega^{-1} a\right) \mathbf{M} .
$$

It can be noted that the Rayleigh damping term presents the attenuation dependent on frequency. It is important to note that the commonly used governing equation of SAFE formulation (2.1) is derived by assuming $k_{z}^{*} \approx k_{z}$; where ${ }^{*}$ denotes complex conjugate [1]. Therefore, the Rayleigh damping model supports just low damping for modes, where $\operatorname{Re}\left(k_{z}\right) \gg \operatorname{Im}\left(k_{z}\right)$.

The loss factor of the waveguide at frequency $\omega$ reads as

$$
\eta=2 \xi=a / \omega+b \omega
$$

where $\xi$ is the damping ratio.

For small to moderate values of damping ratio, the Rayleigh damping and the generalized Maxwell models are equivalent [18] to each other. (3.14) can be linearized and solved as was explained by formulas (3.3) to (3.13). (3.13) provides solutions of $\tilde{k_{L m}}$, therefore $2(2 N+2 M)$ solutions for $k_{z}$ are acquired as

$$
k_{z}= \pm \sqrt{\tilde{k}^{2}-\tilde{k}_{L}^{2}}
$$

The $2 N$ wavenumbers of form $\operatorname{Re}\left(k_{z}\right)+\operatorname{Im}\left(k_{z}\right)$ and $2 N$ wavenumbers of form $-\operatorname{Re}\left(k_{z}\right)-\operatorname{Im}\left(k_{z}\right)$ represent, repectively, outgoing forward and backward wavemodes. The outgoing modes radiate mechanical energy to the surrounding medium while traveling. This energy dissipation causes the decrease of amplitudes characterized by the magnitudes of imaginary part $\left(\operatorname{Im}\left(k_{z}\right) \neq 0\right)$. $2 N$ wavenumbers of form $\operatorname{Re}\left(k_{z}\right)-\operatorname{Im}\left(k_{z}\right)$ and $2 N$ wavenumbers of form $-\operatorname{Re}\left(k_{z}\right)+\operatorname{Im}\left(k_{z}\right)$ represent, respectively, forward and backward incoming modes. Incoming modes represent inward wave from the surrounding medium 
into the waveguide, which amplify the amplitude of the wave within the waveguide. $2 M$ wavenumbers of form $\operatorname{Re}\left(k_{z}\right)+0$ and $2 M$ wavenumbers of form $-R e\left(k_{z}\right)+0$ represent the forward and backward quasi-Scholte wavemodes. They propagate at the waveguide and fluid interface.

Their energy distribution is concentrated along the interface as they travel. Quasi-Scholte waves do not attenuate and disperse much.

In case the Rayleigh damping of immersed waveguide is considered in (3.14), the imaginary parts of the resulting wavenumbers account for energy losses due to geometrical energy spreading and internal friction. The dispersion relations can be presented by phase velocity $c_{p h}=\frac{\omega}{\operatorname{Re}\left(k_{z}\right)}$, which is a ratio between given $\omega$ and real part of calculated wavenumbers $k_{z}$.

\section{Mode tracking}

SAFE models with refined discretization of the waveguide cross-section provide more wavemode solutions to (3.13) due to multi-modal nature of the waves. Different modes with numerically similar wavenumbers for the same angular frequency may appear. Therefore, it is difficult to distinguish the dispersion curves of different relaying just on the graphical appearance of dispersion curves. At different angular frequencies, tracking a mode of an individual modal shape is of interest as the dispersion curves are generated. The approach described in [13] is adopted in this study for tracking the wavenumbers obtained from (3.13) and assigning them to the same mode characterized by its individual modal shape.

Tracking wavenumber $\tilde{k}_{L m}$ at given value $\omega$ is based on finding the increment $\Delta \tilde{k}_{L m}$ at which the updated wavenumber $\tilde{k}_{L m}+\Delta \tilde{k}_{L m}$ at $\omega+\Delta \omega$ would belong to $m$-th mode. Right and left eigen vectors $\left(u_{m}\right.$ and $\left.v_{m}\right)$ of $m$-th mode in (3.12) at frequency $\omega$ satisfy equations

$$
\left(\mathbf{A}^{\prime}-\tilde{k}_{L m} \mathbf{B}^{\prime}\right) \mathbf{u}_{m}=\mathbf{0}, \quad \mathbf{v}_{m}\left(\mathbf{A}^{\prime}-\tilde{k}_{L m} \mathbf{B}^{\prime}\right)=\mathbf{0} .
$$

At frequency $\omega+\Delta \omega(4.1)$ can be rewritten:

$$
\left(\left(\mathbf{A}^{\prime}+\Delta \mathbf{A}^{\prime}\right)-\left(\tilde{k}_{L m}+\Delta \tilde{k}_{L m}\right)\left(\mathbf{B}^{\prime}+\Delta \mathbf{B}^{\prime}\right)\right)\left(\mathbf{u}_{m}+\Delta \mathbf{u}_{m}\right)=\mathbf{0}
$$

where matrices $\Delta A^{\prime}$ and $\Delta B^{\prime}$ are calculated at frequency $\Delta \omega$. After expanding the terms in (4.2) the second order differential terms are omitted for simplicity, therefore $\Delta \omega$ must be selected and varied along $\omega$ with caution. While more sophisticated estimation methods exist (for example, with employment of Pade expansion $[6,9]$, in our case just the linear estimation was sufficient. (4.2) can be simplified to

$$
\left(\mathbf{A}^{\prime}+\tilde{k}_{L m} \mathbf{B}^{\prime}\right) \Delta \mathbf{u}_{m}=\left(\tilde{k}_{L m} \Delta \mathbf{B}^{\prime}+\Delta \tilde{k}_{L m} \mathbf{B}^{\prime}-\Delta \mathbf{A}^{\prime}\right) \mathbf{u}_{m} .
$$

Vector $\Delta \mathbf{u}_{m}$ can be expanded in the base of independent right eigenvectors $\mathbf{u}_{l}(l=\overline{1,2 N+2 M})$ as

$$
\Delta \mathbf{u}_{m}=\sum_{l=1}^{2 N+2 M} \alpha_{l m} \mathbf{u}_{l}
$$


where $\alpha$ is the superposition weight of $l$-th mode for vector $\Delta \mathbf{u}_{m}$ of the tracked $m$-th mode, and $2 N+2 M$ is the number of solutions $\tilde{k}_{L m}$.

Application of orthogonality condition as

$$
\left\{\begin{array}{l}
\mathbf{v}_{m} \mathbf{A}^{\prime} \mathbf{u}_{l}= \begin{cases}\mathbf{v}_{m} \mathbf{A}^{\prime} \mathbf{u}_{m}, & m=l, \\
0, & m \neq l,\end{cases} \\
\mathbf{v}_{m} \mathbf{B}^{\prime} \mathbf{u}_{l}= \begin{cases}\mathbf{v}_{m} \mathbf{B}^{\prime} \mathbf{u}_{m}, & m=l, \\
0, & m \neq l,\end{cases} \\
\tilde{k}_{L m}=\frac{\mathbf{v}_{m} \mathbf{A}^{\prime} \mathbf{u}_{m}}{\mathbf{v}_{m} \mathbf{B}^{\prime} \mathbf{u}_{m}} \\
\end{array}\right.
$$

leads to extraction of the wave number increment from (4.3):

$$
\Delta \tilde{k}_{L m} \approx \frac{\mathbf{v}_{m}\left(\mathbf{A}^{\prime}+\Delta \tilde{k}_{L m} \mathbf{B}^{\prime}\right) \mathbf{u}_{m}}{\mathbf{v}_{m} \mathbf{B}^{\prime} \mathbf{u}_{m}}
$$

This estimate can be used for identifying the wavenumber eigenvalue, which should be chosen for $m$-th dispersion curve at the next angular frequency value.

\section{$5 \quad$ Numerical examples}

The phase velocities of modes of immersed waveguide SAFE model with $3 \times 3$ FE over the rectangular cross-section is presented in (Figure 3(a)).

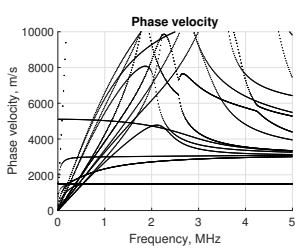

(a)

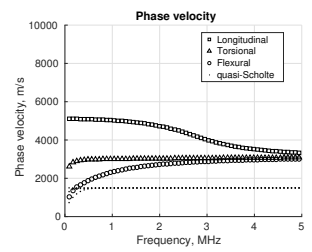

(b1)

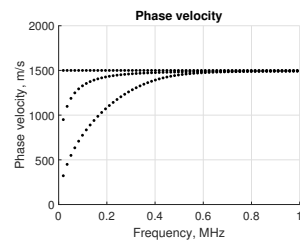

(b2)

Figure 3. (a) Phase velocities of modes in immersed waveguide; (b) phase velocities of fundamental and quasi-Scholte modes in immersed waveguide.

The material properties of the aluminum waveguide are as follows: mass density $m=2780 \mathrm{~kg} / \mathrm{m}^{3}$, Young's modulus $E=7.24 \cdot 10^{10} \mathrm{~Pa}$, Poisson's ratio $\nu=0.34$, cross-section $0.001 \times 0.001 \mathrm{~m}$. The material properties of the perfect fluid (based on water) are as follows: mass density $m=1000 \mathrm{~kg} / \mathrm{m}^{3}$, velocity of pressure (longitudinal) wave $\tilde{c}_{L}=1500 \mathrm{~m} / \mathrm{s}$. The results of tracking only the fundamental and quasi-Scholte modes using (4.4) are shown in (Figure 3(b)). It can be noted that fluid load mostly affects torsional and flexural modes in the low frequency range. However, the longitudinal mode in immersed waveguide does not exhibit evident influence of attenuation at low frequencies, since the displacement field is predominant in $O Z$ direction. Interaction of this mode with fluid at low frequencies is insignificant. The analysis demonstrated that the immersed waveguide of square cross-section supported three distinct groups 
of Scholte waves (zoomed part in Figure 3(b)). The threshold of velocity of these types of modes is $\tilde{c}_{L}=1500 \mathrm{~m} / \mathrm{s}$, which determines them as subsonic waves with respect to the sound velocity in the leaky medium.

The obtained attenuation curves are presented in (Figure 4(a)). The attenuation of fundamental modes is relatively small when compared with the attenuation of higher modes (Figure 4(b)). However, quasi-Scholte modes exhibited low attenuation constant over the frequency range. Therefore, quasi-Scholte modes were almost non-dispersive. They tend to retain their wave shape while travelling, which is a useful feature in many practical applications.

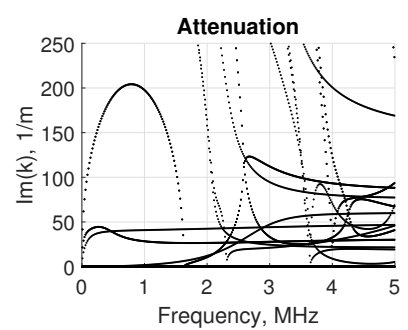

(a)

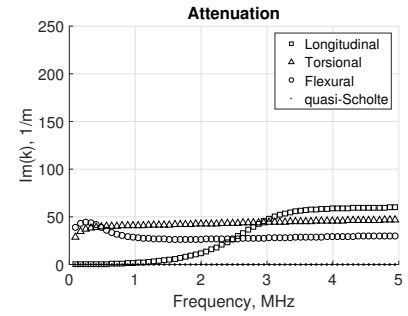

(b)

Figure 4. (a) Attenuation of modes in immersed waveguide; (b) attenuation of fundamental and quasi-Scholte modes in immersed waveguide.

For comparing the obtained dispersion curves results against the dispersion curves exhibited by lossless waveguide surrounded by vacuum, the waveguide with Rayleigh damping and the immersed waveguide was analyzed. Initially, the analysis was carried out by examining a single FE over the cross-section model in order to reveal the main properties of the solutions, which are inherent for the analyzed waveguide. The results are summarized in (Figure 5). For obtaining better precision solutions, further we employ the finer mesh over the of the cross-section.

Figure 5(a) demonstrates that in case of surrounding vacuum three fundamental modes exist at zero frequency. The higher modes appear at cuton frequency, where attenuation $\operatorname{Im}\left(k_{Z}\right)$ approaches zero. Thus, evanescent modes become propagative. Wavenumbers at frequency of $1 \mathrm{MHz}$ (represented by dashed line) on the complex plane come out as symmetric with respect to the axes of the complex plane. The propagative modes correspond to wavenumbers with $\operatorname{Im}\left(k_{Z}\right)=0$, end modes (local vibrations not capable of transferring energy) correspond to $R e\left(k_{Z}\right)=0$ and the remaining modes are evanescent modes with rapidly decaying amplitude. The wavenumbers of form $\pm\left(\operatorname{Re}\left(k_{Z}\right)-\operatorname{Im}\left(k_{Z}\right)\right)$ have no physical meaning as they represent fictitious wavemodes with increasing amplitude as they travel.

In case the damping is present, (Figure 5(b)), all the modes are evanescent. Their wavenumbers do not retain symmetry on the complex plane and the cuton frequencies do not apply anymore. At higher frequencies in case of severe damping (for example, $a=1 \cdot 10^{6}, b=3 \cdot 10^{-8}$ ) the phase velocity curve does not converge to the phase velocity of Rayleigh's surface wave. This indicates 


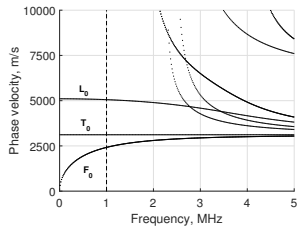

(a1)

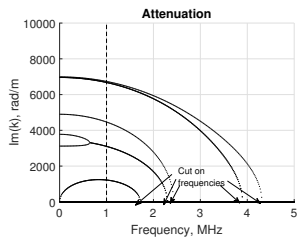

(a2)

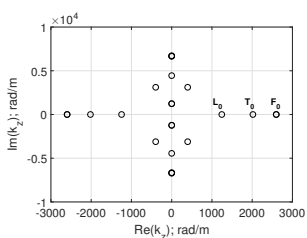

(a3)

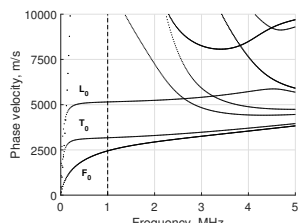

(b1)

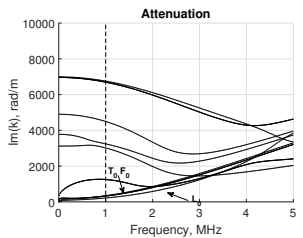

(b2)

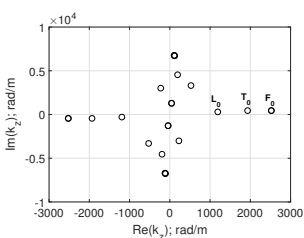

(b3)

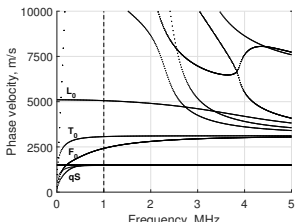

$(\mathrm{c} 1)$

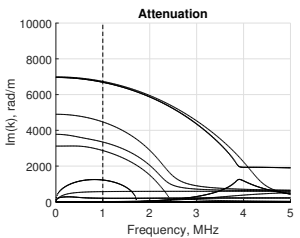

$(\mathrm{c} 2)$

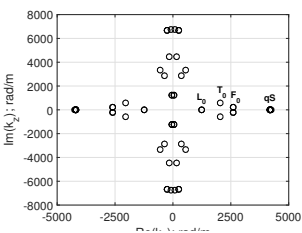

(c3)

Figure 5. Phase velocity (1), attenuation (2) and wavenumbers (3; at $1 \mathrm{MHz}$ (dashed line)) of waveguide in vacuum (a); with Rayleigh damping $a=1 \cdot 10^{6}, b=3 \cdot 10^{-8}$ (b); immersed in water (c).

the appearance of certain errors of wave solutions, which are caused by the commonly used assumption $k_{Z}^{*}=k_{Z}$ in case of some more damped waveguides.

In case of the immersed waveguide (Figure 5(c)) all the modes (with exception of quasi-Scholte modes) are evanescent. Some modes have cut on frequencies the same as in case of being surrounded by vacuum instead, and the wavenumbers demonstrate symmetry with respect to the axes of the complex plane.

The dispersion results for fundamental modes of immersed waveguide with Rayleigh damping, where coefficients $a$ and $b$ have been varied, are shown in (Figure 6). As damping was increased, quasi-Scholte wavemode remained unattenuated and merged to the common group of wavemodes with constant phase velocity. In case of severe damping (Figure 6(c)) the phase velocity curves of fundamental modes at higher frequencies tended to bend away from the dispersion curves of quasi-Scholte modes. This clearly indicates non-feasible results, and the symmetry of wavenumbers on complex plane is destroyed. The cause of the inadequacy of the damped immersed waveguide model is likely due to the fact, that assumption about complex conjugate of the wavenumbers was as previously no longer met. In this analysis the model is considered to be adequate as long phase velocities asymptotically and monotonically approach Rayleigh 
surface wave phase velocity value. This is valid for isotropic waveguides [19].

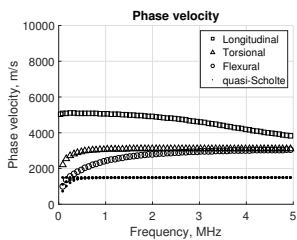

(a1)

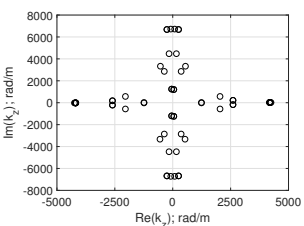

(a2)

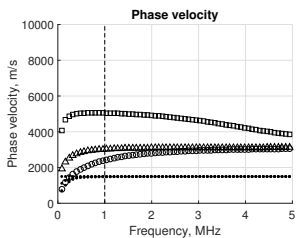

(b1)

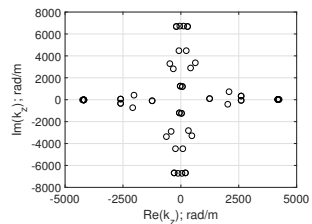

(b2)

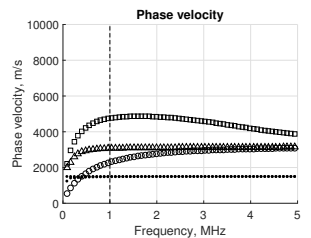

(c1)

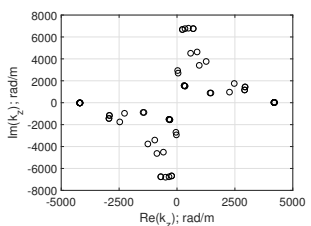

$(\mathrm{c} 2)$

Figure 6. Phase velocity (1) and wavenumbers (2; at $1 \mathrm{MHz}$ (dashed line)) of immersed waveguide with Rayleigh damping; $a=1 \cdot 10^{5}, b=1 \cdot 10^{-10}$ (a); $a=1 \cdot 10^{6}, b=1 \cdot 10^{-9}$ (b); $a=1 \cdot 10^{7}, b=1 \cdot 10^{-8}$ (c).

The verification of the model has been performed by using the numerical experiments where the waveguide had cross-section of $0.006 \times 0.012 \mathrm{~m}$, FE mesh was $3 \times 6$. Initially, the ratio between the height and length of the cross-section is $1: 2$. As this ratio increases, the obtained dispersion curves converge to those of the plate (Figure 7).

As the longitudinal mode at close to zero frequency approaches the threshold of wave velocity in the plate as $c_{\text {plate }}=\frac{\sqrt{E}}{\sqrt{\rho\left(1-\nu^{2}\right)}}($ dashed line in Figure 7$)$ it can be assumed that the obtained fundamental modes of the analyzed waveguide match those of the plate of the same height as the height of the cross-section of the waveguide. The results show evident convergence and reasonably well agree with results published in [11] for the plate.

\section{Conclusions}

This study deals with the different techniques of acquiring dispersion relations for damped three-dimensional waveguide immersed in the perfect fluid, as well as with the analysis of the obtained wavemodes. This enables further investigation of quasi-Scholte waves. Up to date three-dimensional quasi-Scholte modes haven't been given much attention yet. The coupling of two attenuation mechanisms due to energy leakage and material damping has shown that the models of immersed waveguide used in this study support only weak and moderate Rayleigh damping. Adequacy of the model supporting solutions for more severe damping also requires consideration of the complex conjugate of the wavenumber in the governing finite element formulation, which would lead to non-linear eigenproblems. 


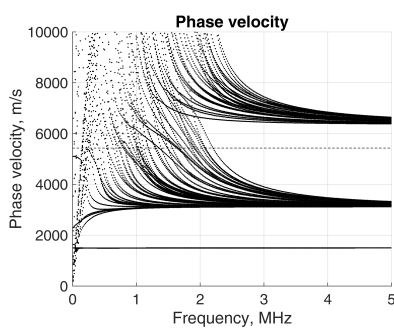

(a)

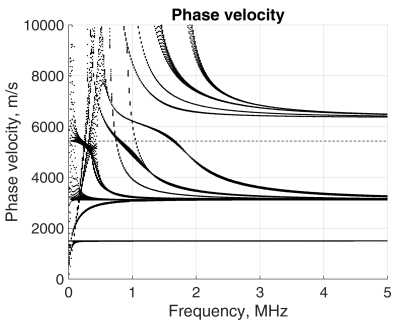

(c)

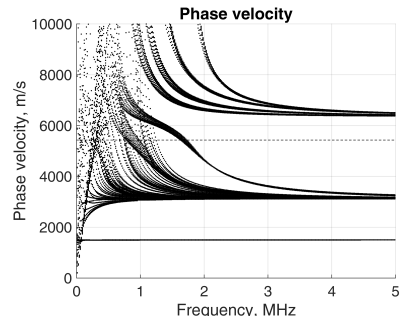

(b)

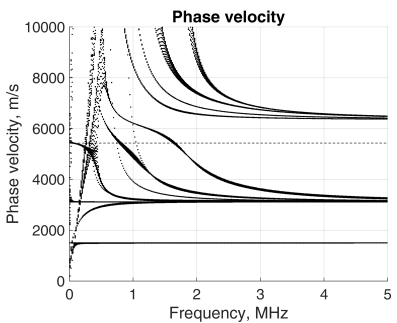

(d)

Figure 7. The convergence of the model for cases (a) mesh $3 \times 6$ and cross-section size ratio $1: 2$, (b) mesh $3 \times 10$ and cross-section size ratio $1: 10$, (c) mesh $3 \times 15$ and cross-section size ratio $1: 100$, (d) mesh $3 \times 15$ and cross-section size ratio $1: 1000$.

The future directions of this research include derivation of group velocity, force response and further explorations on effects of Rayleigh damping.

\section{References}

[1] R. Barauskas, A. Nečiūnas and M. Patašius. Elastic wave simulation based on modal excitation in 3D medium. Journal of Vibroengineering, 18(8), 2016. https://doi.org/10.21595/jve.2016.18101.

[2] I. Bartoli, A. Marzani, F. Lanza di S. and E. Viola. Modeling wave propagation in damped waveguides of arbitrary cross-section. Journal of Sound and Vibration, 295(3-5):685-707, 2006. ISSN 0022-460X. https://doi.org/10.1016/j.jsv.2006.01.021.

[3] M. Bilodeau, N. Quaegebeur and P. Masson. Design of a guided wave absorber for Structural Health Monitoring system development. $\quad N D T$ \& $E$ International, 88:33-41, 2017. ISSN 0963-8695. https://doi.org/10.1016/j.ndteint.2017.03.003.

[4] M. Castaings and M. Lowe. Finite element model for waves guided along solid systems of arbitrary section coupled to infinite solid media. The Journal of the Acoustical Society of America, 123(2):696-708, February 2008. ISSN 00014966. https://doi.org/10.1121/1.2821973. Available from Internet: 10.1121/1 . 2821973.

[5] K.F. Graff. Wave motion in elastic solids. Courier Corporation, 2012. 
[6] H. Gravenkamp, C. Birk and C. Song. Computation of dispersion curves for embedded waveguides using a dashpot boundary condition. The Journal of the Acoustical Society of America, 135(3):1127-1138, 2014. https://doi.org/10.1121/1.4864303.

[7] H. Gravenkamp, C. Birk and C. Song. Numerical modeling of elastic waveguides coupled to infinite fluid media using exact boundary conditions. Comput. Struct., 141:36-45, August 2014. ISSN 0045-7949. https://doi.org/10.1016/j.compstruc.2014.05.010. Available from Internet: 10. 1016/j. compstruc. 2014.05.010.

[8] H. Gravenkamp, C. Birk and J. Van. Modeling ultrasonic waves in elastic waveguides of arbitrary cross-section embedded in infinite solid medium. Comput. Struct., 149:61-71, March 2015. ISSN 0045-7949. https://doi.org/10.1016/j.compstruc.2014.11.007.

[9] H. Gravenkamp, H. Man, C. Song and J. Prager. The computation of dispersion relations for three-dimensional elastic waveguides using the Scaled Boundary Finite Element Method. Journal of Sound and Vibration, 332(15):3756-3771, 2013. ISSN 0022-460X. https://doi.org/10.1016/j.jsv.2013.02.007.

[10] H. Gravenkamp, C. Song and J. Prager. A numerical approach for the computation of dispersion relations for plate structures using the Scaled Boundary Finite Element Method. Journal of Sound and Vibration, 331(11):2543-2557, 2012. ISSN 0022-460X. https://doi.org/10.1016/j.jsv.2012.01.029.

[11] T. Hayashi and D. Inoue. Calculation of leaky Lamb waves with a semianalytical finite element method. Ultrasonics, 54(6):1460-1469, aug 2014. https://doi.org/10.1016/j.ultras.2014.04.021.

[12] T. Hayashi, W.-J. Song and J.L. Rose. Guided wave dispersion curves for a bar with an arbitrary cross-section, a rod and rail example. Ultrasonics, 41(3):175183, 2003. ISSN 0041-624X. https://doi.org/10.1016/S0041-624X(03)00097-0. Available from Internet: http://www.sciencedirect.com/science/article/ pii/S0041624X03000970.

[13] T. Hayashi, W.J. Song and J.L. Rose. Guided wave dispersion curves for a bar with an arbitrary cross-section, a rod and rail example. Ultrasonics, 41(3):175183, May 2003. ISSN 0041-624X. https://doi.org/10.1016/s0041-624x(03)000970.

[14] M. Mazzotti, I. Bartoli, A. Marzani and E. Viola. A coupled SAFE-2.5D BEM approach for the dispersion analysis of damped leaky guided waves in embedded waveguides of arbitrary cross-section. Ultrasonics, 53(7):1227-1241, 2013. ISSN 0041-624X. https://doi.org/10.1016/j.ultras.2013.03.003.

[15] A.H. Nayfeh and P.B. Nagy. Excess attenuation of leaky Lamb waves due to viscous fluid loading. The Journal of the Acoustical Society of America, 101(5):2649-2658, 1997. https://doi.org/10.1121/1.418506.

[16] F.H. Quintanilla, Z. Fan, M.J.S. Lowe and R.V. Craster. Guided waves' dispersion curves in anisotropic viscoelastic single-and multi-layered media. In Proc. R. Soc. A, volume 471, p. 20150268. The Royal Society, 2015.

[17] J.M. Renno and B.R. Mace. On the forced response of waveguides using the wave and finite element method. Journal of Sound and Vibration, 329(26):5474-5488, 2010. ISSN 0022-460X. https://doi.org/10.1016/j.jsv.2010.07.009.

[18] J.-F. Semblat. Rheological interpretation of Rayleigh damping. arXiv preprint arXiv:0901.3717, 2009. 
[19] D. O. Thompson and D. E. Chimenti. Preface: Review of progress in quantitative nondestructive evaluation. AIP Conference Proceedings, 1430(1):35, 2012. https://doi.org/10.1063/1.4716208. Available from Internet: http: //aip.scitation.org/doi/abs/10.1063/1.4716208.

[20] F. Treyssede. Spectral element computation of high-frequency leaky modes in three-dimensional solid waveguides. Journal of Computational Physics, 314:341354, 2016. https://doi.org/10.1016/j.jcp.2016.03.029.

[21] F. Treyssede and L. Laguerre. Numerical and analytical calculation of modal excitability for elastic wave generation in lossy waveguides. Journal of the Acoustical Society of America, 113:827-3837, 2014.

[22] I.A. Viktorov. Rayleigh and Lamb waves: physical theory and applications. Plenum press, 1967.

[23] E. Viola and A. Marzani. Mechanical Vibration: Where do we Stand?, volume 488 of International Centre for Mechanical Sciences, chapter Exact Analysis of Wave Motions in Rods and Hollow Cylinders, pp. 83-104. Springer Vienna, 2007. ISBN 978-3-211-68586-0.

[24] O.C. Zienkiewicz, R.L. Taylor, O.C. Zienkiewicz and R.L. Taylor. The finite element method, volume 3. McGraw-hill London, 1977.

\section{Appendix}

The amplitudes of the wave displacement field in fluid at arbitrary $x_{a r b}$ above the waveguide side $\Gamma 1$ (Figure 1(b)) is the two-dimensional wave

$$
\tilde{\mathbf{u}}_{\text {fluid } 1}=\left(\begin{array}{l}
\tilde{u}_{y} \\
\tilde{u}_{z}
\end{array}\right)=\left(\begin{array}{l}
\tilde{k}_{L} \\
\tilde{k}_{z}
\end{array}\right) a_{L} e^{i\left(\tilde{k}_{L} y+k_{z} z\right)},
$$

where $a_{L}$ is an arbitrary scalar.

The amplitude of the resulting wave depends on wavenumbers of both waves. Since excited wave in leaky medium is longitudinal and perpendicular to $\Gamma 1$, displacements $\tilde{u}_{x}=0$. The displacement field for leaky wave on boundary $\Gamma 1$ at $z=0$ and $x_{a r b}$ is:

$$
\tilde{\mathbf{u}}_{\Gamma 1}=\left(\begin{array}{c}
u_{n} \\
u_{z}
\end{array}\right)=\left(\begin{array}{c}
\tilde{u}_{n} \\
\tilde{u}_{z}
\end{array}\right)=\left(\begin{array}{c}
\tilde{k}_{L} \\
\tilde{k}_{z}
\end{array}\right) a_{L} e^{i \tilde{k}_{L} y_{1}} .
$$

Scalar $a_{L}$ can be then expressed as

$$
a_{L}=\frac{u_{n}}{\tilde{k}_{L}} e^{-i \tilde{k}_{L} y_{1}}
$$

Then the (6.1) can be rewritten:

$$
\tilde{\mathbf{u}}_{\text {fluid } 1}=\left(\begin{array}{c}
\tilde{u}_{y} \\
\tilde{u}_{z}
\end{array}\right)=\left(\begin{array}{c}
1 \\
\tilde{k}_{z} / \tilde{k}_{L}
\end{array}\right) u_{n} e^{i\left(\tilde{k}_{L}\left(y-y_{1}\right)+k_{Z} z\right)} .
$$

The strain vector for leaky wave in fluid affected by boundary $\Gamma 1$ is

$$
\tilde{\epsilon}_{f l u i d 1}=\left(\mathbf{L}_{y} \frac{\partial}{\partial y}+\mathbf{L}_{z} \frac{\partial}{\partial z}\right) \tilde{\mathbf{u}}_{\text {fluid } 1}=\left(i \tilde{k}_{L} \mathbf{L}_{y}+i k_{z} \mathbf{L}_{z}\right)\left(\begin{array}{c}
1 \\
\tilde{k}_{z} \\
\tilde{k}_{L}
\end{array}\right) u_{n} e^{i\left(\tilde{k}_{L}\left(y-y_{1}\right)+k_{z} z\right)},
$$


where $\mathbf{L}_{y}=\left(\begin{array}{ll}1 & 0 \\ 0 & 0 \\ 0 & 1\end{array}\right)$ and $\mathbf{L}_{z}=\left(\begin{array}{ll}0 & 0 \\ 0 & 1 \\ 1 & 0\end{array}\right)$.

The strain vector for leaky wave on boundary $\Gamma 1$ is:

$$
\tilde{\epsilon}_{\Gamma 1}=\left(\begin{array}{c}
\tilde{\epsilon}_{y y} \\
\tilde{\epsilon}_{z z} \\
\tilde{\gamma}_{y z}
\end{array}\right)=\left(i \tilde{k}_{L} \mathbf{L}_{y}+i k_{z} \mathbf{L}_{z}\right)\left(\begin{array}{c}
1 \\
\tilde{k}_{z} \\
\tilde{k}_{L}
\end{array}\right) u_{n} e^{i k_{z} z}
$$

However, the stress on boundary $\Gamma 1$ is present only in direction of displacements $u_{n}$ and can be expressed using projection $\tilde{k}_{L}=\sqrt{\tilde{k}^{2}-\tilde{k}_{Z}^{2}}$ of the wavenumber vector $\tilde{\mathbf{k}}$ :

$$
\tilde{\sigma}_{\Gamma 1}=\left(\begin{array}{c}
\tilde{\sigma}_{y y \Gamma 1} \\
\tilde{\sigma}_{z z \Gamma 1} \\
\tilde{\tau}_{z y \Gamma 1}
\end{array}\right)=\left(\begin{array}{c}
\tilde{\sigma}_{y y \Gamma 1} \\
0 \\
0
\end{array}\right)
$$

where

$$
\tilde{\sigma}_{y y \Gamma 1}=-i \tilde{\rho} \tilde{c}_{L} \omega \tilde{u}\left(\tilde{k}_{L}, y=y 1, z\right)=-i \tilde{\rho} \frac{\omega}{\sqrt{\tilde{k}^{2}-\tilde{k}_{Z}^{2}}} \omega \tilde{u}_{y}=-i \tilde{\rho} \frac{\omega^{2}}{\tilde{k}_{L}} \tilde{u}_{y} .
$$

Using (6.2) $\tilde{\sigma}_{y y \Gamma 1}$ can be rewritten:

$$
\tilde{\sigma}_{y y \Gamma 1}=-i \tilde{\rho} \frac{\omega^{2}}{\tilde{k}_{L}} \tilde{u}_{y}=-i \tilde{\rho} \frac{\omega^{2}}{\tilde{k}_{L}} \tilde{u}_{n} e^{i k_{Z} z} .
$$

Assuming the continuity for stresses at the boundary of solid and fluid, the stress on boundary $\Gamma 1$ in fluid corresponds to surface traction condition for waveguide as

$$
\mathbf{t}_{\Gamma 1}=\left(t_{n}, t_{\tau}, t_{z}\right)^{T}=\left(t_{n}, 0,0\right)^{T} .
$$

It can be noted, that the stress in the fluid at the interface may be regarded as surface stress applied on the waveguide. Both stress in fluid and interface of waveguide along $O Z$ direction is governed by harmonic wave:

$$
\mathbf{t}_{\Gamma 1}=\left(t_{n}, t_{\tau}, t_{z}\right)^{T}=\mathbf{N} \mathbf{T}_{j} e^{i k_{Z} z},
$$

where $\mathbf{T}_{j}$ is nodal external traction vector on $\Gamma 1$. 